\title{
A protocol study of novice interaction design behaviour in Botswana: solution-driven interaction design
}

\author{
Helen Sharp, Nicole Lotz, Richard Blyth, Mark Woodroffe \\ The Open University, \\ Walton Hall, Milton Keynes, MK7 6AA, UK \\ \{helen.sharp; nicole.lotz; richard.blyth; \\ mark.woodroffe\}@open.ac.uk
}

\author{
Dino Rajah, Turugare Ranganai \\ Botho University, Botho Education Park, Kgale \\ PO Box 501564, Gaborone, Botswana \\ \{dino.rajah; turugare.ranganai\}@bothouniversity.ac.bw
}

\begin{abstract}
Think aloud studies and protocol analysis are well-known in the field of $\mathrm{HCl}$, but most often these studies focus on usability evaluations, or on the use of technology. Rarely are they used to investigate the behaviour of interaction designers. In this paper, we report on a protocol study with novice interaction designers in Botswana. Participants had just completed the design section of an undergraduate module on Interaction Design that actively promotes a problem-driven approach to the design of interactive products, yet the participants behaved in a way that is closer to a solution-driven approach. The module emphasizes user-centred design, prototyping methods to support design development, and evaluating design detail. Yet participants suggest solutions before exploring the context of use, use prototyping methods to capture, rather than to develop, designs, and do not produce detailed designs. In a problem-solving context, some of these behaviours are typical of novices, but in a design context they are also seen in experienced designers. The results presented here reveal the detail of the approach adopted by these students, and contribute to the wider debate concerning the internationalization of $\mathrm{HCl}$ education.
\end{abstract}

novice behaviour, protocol analysis, design studies, cultural influences

\section{INTRODUCTION}

Many protocol studies in $\mathrm{HCl}$ are used to evaluate usability or the use of technology, e.g. van den Haak et al (2004), Oyugi et al (2008). Rarely are they used to investigate interaction design itself. On the other hand, design studies regularly use protocol analysis to gain insights into the behaviour of novice and expert designers, e.g. Gero and Tang (2001), Coley et al (2007), McDonnell and Lloyd (2009). If educators and trainers have insight into how novice designers are approaching a design problem, then they will be better able to guide and support them in their journey towards becoming an expert. The protocol study presented here takes one step towards this goal.

This particular study was prompted by the observation that African-based students studying a module in Interaction Design seemed to be taking a very different approach to design problems than the module materials encouraged. The teaching materials for the module are very detailed and prescriptive, with the weekly materials, exercises, examples and assessments all being set out in printed books that structure and enhance the teaching in the core text book (Sharp et al, 2007).
Local tutors had been briefed on module delivery and observed for quality assurance, yet the variation in design behaviour persisted over several years. This investigation of students' behaviours was therefore instigated to understand what our Botswana students are actually doing, in order to support their learning more appropriately.

This work takes place in a context where cultural influences on software development, design and education are under scrutiny, and the approach to education and design in a global setting is being questioned. For example Fendler and WinchiersTheophilus (2010) suggest that software engineering culture is heavily influenced by western culture, and propose that software engineering education be modified for the local situation. In addition, Smith et al (2007) point out that principles underlying the user-centred approach to software development, which is central to much $\mathrm{HCl}$ education, are derived from USA and Northern Europe.

The globalization of product and software development has resulted in calls for processes and methods to be tailored for the local situation (e.g. Chavan, 2005). Smith et al (2010) suggest 
that localisation of methods to suit local situations is needed, not just a set of Western case studies, and that an education in $\mathrm{HCl}$ methods rather than tools training is needed.

So how do novice interaction designers from Africa tackle an interaction design problem? How do they adopt and apply the methods and approaches taught? Answers to these questions contribute to discussions in global software development, education and design.

The next section introduces the background to this study. Section 3 describes the study design: data collection and analysis. Section 4 presents the findings and section 5 discusses them in the context of relevant literature, local African perspectives, and the taught module. Section 6 identifies limitations to the study and section 7 concludes the paper.

\section{BACKGROUND}

\subsection{The Interaction Design module}

The module in question is a distance education module offered by the UK's Open University. The module is based on a well-known $\mathrm{HCl}$ textbook Interaction Design (Sharp et al, 2007) and includes detailed wrap-around material to guide and support students' learning. The module promotes the definition of usability and user experience goals, exploring the problem space and challenging any underlying assumptions before starting to produce a solution.

This module is offered by Botho College in Botswana. Botho College is a private tertiary institution registered with the Tertiary Education Council (www.tec.org.bw) and the Botswana Training Authority (www.bota.org.bw). The institution has been offering IT programmes since its inception in 1997. The institution operates in three locations in Botswana: Gaborone, Francistown and Maun. The three locations together have around 4,000 students. The study reported here was conducted in Francistown.

\subsection{Culture, Interaction Design and global development}

Several studies provide evidence that cultural factors influence both the design of a product (e.g. Moalosi et al, 2007) and the process of design (e.g. Marsden et al, 2008). For example, Faiola and Matei (2005) found that website users performed better on tasks when the website was designed by people from the same culture, indicating that the culture impacts upon the design product; Razzaghi et al, (2009) identified patterns of cultural variations in novices' sketches of design concepts and Gautam and Blessing (2010) found differences in the design process between industrial designers from Germany, China and India.
These studies did not cover interaction design, but Smith et al (2010) argue that cultural differences have the potential to affect both the product and the process of Interaction Design as well, and they call for a holistic understanding of $\mathrm{HCl}$ within the local practitioner community, and localization of methods and tools to meet local requirements. Fendler and Winchiers-Theophilus (2010) have highlighted this issue in software engineering and propose a framework that challenges the notion of "universally transferable" education and takes account of specific software development contexts. Their work emphasises that the way forward is to examine a range of methods and knowledge to assess similarities, differences and local influential factors rather than to take 'western' approaches and tailor them to developing nations. We would argue that a key aspect of such an approach is understanding the detailed behaviour of local students, which may have developed through previous education experiences, or from other socio-cultural factors.

\subsection{Design behaviours, design thinking and interaction design}

There is a long history of studies that have investigated designer behaviour and design thinking. Initially there was an emphasis on problem-solving behaviour (e.g. Chi et al, 1988), but as Dorst (2003) points out, although some aspects of design involve problem-solving, not all of design is like that. The differences between novice and expert design behaviour have been a particular focus of study. Cross (2004) summarises the studies on expertise, and comments that there is still considerable work to be done to establish a robust and reliable understanding of expertise in design. He also warns that problem-solving expertise seems often to be contradicted by the behaviour of expert designers, and that expertise is often not transferrable between domains.

Taking a process view of design, Kruger and Cross (2006) identify four design strategies: problemdriven, solution-driven, information-driven and knowledge-driven. In the first, designers focus on defining the problem, in the second they emphasise generating solutions, the third focuses on gathering information and the fourth uses the designer's personal knowledge.

Little work has been done to investigate interaction design behaviour, although Saffer (2010) identifies four different approaches to interaction design and acknowledges that different design tasks relate best to different approaches: genius, user-centred, activity-centred, systems design. Visser (2006), after reviewing design in the domain of $\mathrm{HCl}$ writes, "Is there specificity to $\mathrm{HCl}$ design and what is it?" She goes on to suggest that design is multi-faceted and that characterising design as problem-solving does not capture its essence. Instead she proposes a view of design that focuses on construction of 
representations. So are interaction designers likely to behave like designers or problem-solvers? The picture is not clear, but it is likely that they will engage in a bit of both. One distinctive feature of interaction design when compared to design more generally is the need to design explicitly for interaction with technology; another is the interdisciplinary nature of Interaction Design.

\section{CONSTRAINED TASK STUDY DESIGN}

The study was designed to address one main research question:

What patterns of behaviour are exhibited by novice interaction designers in Botswana, i.e. what are Botswana students doing when they tackle an interaction design problem?

To address this question, a constrained task study was designed and executed.

\subsection{Constructive interaction protocol}

Verbalisation and subsequent protocol analysis is routinely used in design studies, and according to Coley et al (2007) is, "the most popular method in design research for the capturing, understanding, and analysis of design thinking".

In our study in Botswana we decided to use the method of constructive interaction to elicit verbal protocols (O'Malley et al, 1985; Miyake, 1986). This involves paired participants instructed to collaborate on a task and talk about what they are doing and thinking. Some of the problems of concurrent protocols such as silence and inhibition can be overcome by constructive interaction. Constructive interaction also avoids the possible cultural influences on concurrent protocol verbalisation (Clemmensen et al, 2008) and the focus on design product rather than process, which is a concern of retrospective protocols (Kuusela and Paul, 2000). The decision not to use concurrent protocols also aligns with a personal communication from Winschiers-Theophilus that concurrent protocols would not be effective with African students.

\subsection{Study procedure}

Participants were invited to take part in a protocol study in which they were video and audio recorded. Each session lasted around two hours, including instructions and warm-up activity, with one hour being dedicated to the main design task. Participants were asked which language they would prefer to use, and were paired according to that choice. To reduce any possible inhibition, the study was run by a facilitator who spoke the chosen language, and the participant booklet was translated into their language of choice. A facilitator guide with step-by-step procedures was provided and the researchers watched the session from another room through a CCTV link. The materials provided to participants were: module books, design method summaries (usability and user experience goals, scenarios, storyboards and interface sketches), paper, pencils, refreshments, and a participant booklet each. The participant booklet contained the following sections:

- Study background

- Consent form

- Warm-up activity (Towers of Hanoi)

- Design brief: a medication system for homebased patients

A diagram of the room layout is shown in Figure 1.

Participants were asked to design a new interactive product that will help ensure sick people staying at home take the right drug at the right time. The product was to be usable by the young and elderly.

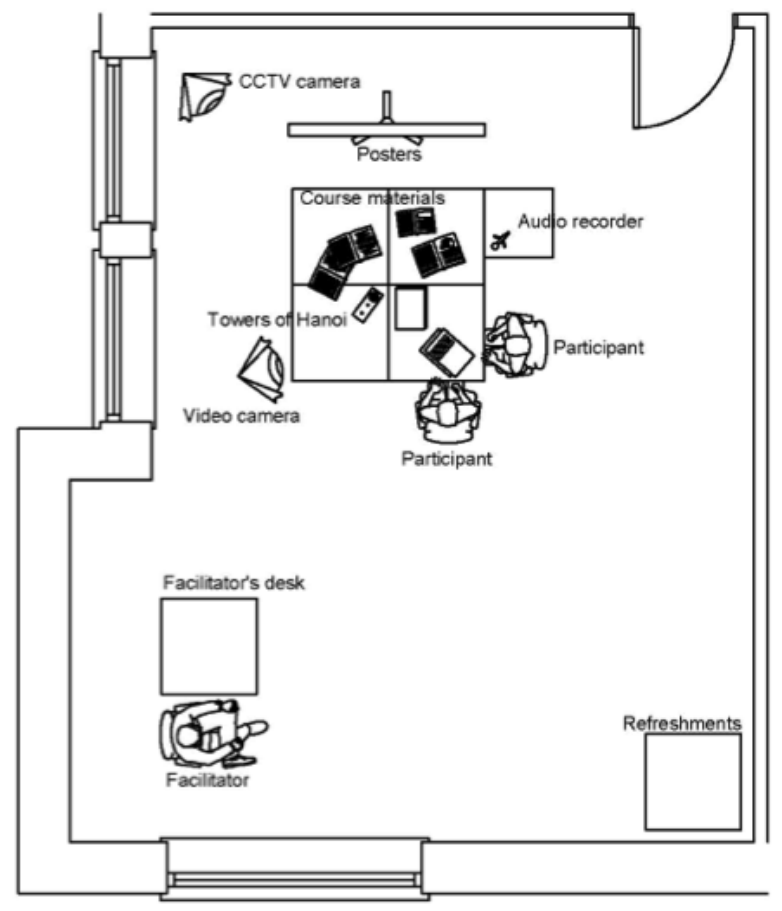

Figure 1: The room layout for the constrained task study

\subsection{Data analysis}

Audio and video data were translated and transcribed by local interpreters, and the translation was superimposed on the video. The analysis reported here focuses on the English transcripts.

The transcripts were analysed using a modified and extended version of Valkenburg and Dorst (1998)'s notation to identify the processes in Schön (1983)'s design and reflection cycle: naming, framing, moving and reflecting. The extended version includes signature frame matrices to more clearly identify frames (Blyth et al, 2012), three functions of analogies (Christensen and Schunn, 2007), and a more detailed notation that highlights the distinction between thinking in the problem space and in the solution space (Lotz et al, 2013). The coding was 
completed by two researchers independently and challenged by two others on a regular basis.

Through this analysis we were looking for interesting patterns of behaviour. In this context, a behaviour may be a 'pattern' whether it's seen many times in one session, with the same participants, or across sessions with different participants.

\subsection{Participants}

Thirty participants were chosen from a volunteer set of 70. All participants were studying the Interaction Design module described above, and had just completed the design section of the module. This resulted in 15 sessions; data from two of the sessions was unusable because the protocols were too quiet and another one was unusable because of 'leading' facilitator instructions. Participants were paired according to several factors including assessment scores, rural or urban background and preferred language.

Pairs 4 and 6 chose to conduct the session in Kalanga, while all other pairs chose Setswana. Twenty-five participants were female and five were male, which broadly reflects the female:male ratio across the module. Half of the participants were under 24, and the ages ranged from 21 to over 30 . All participants had an average assessment score on this module of over $50 \%$, and all of them owned a mobile phone. Ownership of a computer, a radio and a television was also evident but not comprehensive. Nearly all the students were from government or community schools and most of them had been exposed to some design - mainly art, design and technology, and fashion and fabrics.

\section{FINDINGS}

Several behaviours emerged from our analysis of the constrained task (see Table 1). In this paper we will consider the following seven patterns.

1 Participants immediately suggest a solution when they are given a design problem.

2 Participants use design methods to represent design solutions.

3 When participants approach a design problem the solutions they come up with are not very detailed.

$4 \quad$ Participants produce multiple outline solutions.

5 Participants often change direction, one moment talking about one solution and the next about another solution.

6 Participants use analogy to help set their design direction.

7 Participants generate adaptations of simple, practical, off the shelf designs.

Examples of each of these behaviours are included below. In section 4.8 the overall design strategy is considered.

\subsection{Participants immediately suggest a solution when they are given a design problem}

A pattern shown in 8 of the 12 sessions was for participants to offer a solution within the first minute of designing after the facilitator had withdrawn. For example "let's design a watch". Two example transcripts illustrating this behaviour are shown below.

Table 1 Overall view of pairs exhibiting the identified behaviours

\begin{tabular}{|c|c|c|c|c|c|c|c|}
\hline Pair & $\begin{array}{l}\text { Solution } \\
\text { first }\end{array}$ & $\begin{array}{l}\text { Methods } \\
\text { represent } \\
\text { solutions }\end{array}$ & $\begin{array}{l}\text { Lack of } \\
\text { detail }\end{array}$ & $\begin{array}{l}\text { Many } \\
\text { outline } \\
\text { solutions }\end{array}$ & $\begin{array}{l}\text { Change } \\
\text { direction }\end{array}$ & $\begin{array}{l}\text { Analogies } \\
\text { used } \\
\text { (number) }\end{array}$ & $\begin{array}{l}\text { Simple off- } \\
\text { the-shelf } \\
\text { designs }\end{array}$ \\
\hline 1 & $x$ & $x$ & $\checkmark$ & $x$ & $x$ & 3 & $\checkmark$ \\
\hline 2 & $\checkmark$ & $x$ & $\checkmark$ & $\begin{array}{l}\text { 5: alarm, } \\
\text { system, } \\
\text { watch, cell, } \\
\text { wallclock }\end{array}$ & $\checkmark$ & 5 & $\checkmark$ \\
\hline 3 & $\checkmark$ & $\checkmark$ & $\checkmark$ & $\begin{array}{l}\text { 2: cell and } \\
\text { volunteer }\end{array}$ & $\checkmark$ & 3 & $\checkmark$ \\
\hline 4 & $\checkmark$ & $x$ & $x$ & $\mathrm{x}$ & $x$ & 7 & $x$ \\
\hline 5 & $\checkmark$ & $\checkmark$ & $\checkmark$ & $\begin{array}{l}\text { 2: cell and } \\
\text { watch }\end{array}$ & $\checkmark$ & 2 & $\checkmark$ \\
\hline 6 & $\checkmark$ & $x$ & $x$ & $\mathrm{x}$ & $\checkmark$ & 4 & $\checkmark$ \\
\hline 7 & $x$ & $x$ & $x$ & $x$ & $x$ & 4 & $\checkmark$ \\
\hline 8 & $\checkmark$ & $\checkmark$ & $\checkmark$ & $\begin{array}{l}\text { 2: watch, } \\
\text { phone }\end{array}$ & $\checkmark$ & 2 & $\checkmark$ \\
\hline 9 & $x$ & $\checkmark$ & $x$ & $\mathrm{x}$ & $x$ & 2 & $x$ \\
\hline 10 & $\checkmark$ & $\checkmark$ & $\checkmark$ & $x$ & $x$ & 4 & $\checkmark$ \\
\hline 12 & $\checkmark$ & $\checkmark$ & $\checkmark$ & $\begin{array}{l}\text { 2: phone, } \\
\text { training }\end{array}$ & $\checkmark$ & 1 & $\checkmark$ \\
\hline 13 & $x$ & $x$ & $x$ & $\mathrm{x}$ & $x$ & 2 & $\checkmark$ \\
\hline
\end{tabular}




\section{Pair 6}

A. What are we doing?

B. Something with an alarm...?

A. I do not understand.

B. Now the product has to be used by a patient and a doctor. We can start by sketching it and explain it later. How it is going to be used? So you have any idea. A watch

A. We design what sort of an object? Let's design a watch.

B. What sort of watch?

A. A watch with voice recognition, it would be informing patients when its time is up and for them to take their medication or let's design a television. A television will show motion pictures so that if you cannot read you will see the pictures and those would only play when its medication time, a short scene with an actor, performing as if $\mathrm{s} / \mathrm{he}$ is taking their medication.

\section{Pair 9}

A. This solution needs an interactive design for us to come up with the proposed product. We can have it designed by doctors and used by patients at home. Doctors will give a prescription depending on the agreement between him and a patient and a doctor is responsible for configuring the times for taking medication.

B. Yes

A. Product will have two buttons, red and green button. Green will be indicating time to take medication. Do you get the logic?

B. Yes I do

A. This proposed product will work just like a cellphone. Red button indicates that the time has passed and audio for alerting a patient that it is time up. It should also include handles and a stand

B. Handles and a stand so that it can support its own weight

\subsection{Participants use design methods to represent (capture) design solutions}

Although most pairs mention design methods such as storyboards or scenarios early in their designing, they don't use the methods to develop the solution, but use them to capture a solution. For example, Pair 9 talk to each other about the watch design (Figure 2a) before sketching it. While producing the sketch they focus on both details of the design, such as colour of buttons, and the method itself, i.e. what is an interface sketch for. Pair 10 sketch the wrist band (Figure 2b) and then ask "so which design method shall we use? A storyboard?", indicating directly that they would not use the storyboard to develop the design.

Pair 2 explicitly state that this is what they will do: "We draft all our ideas and then transfer the best one into our storyboard." Their storyboard is not begun until over half way through the session. (a)

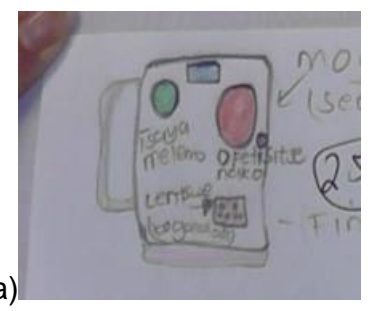

(b)

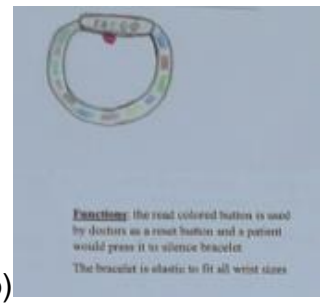

Figure 2: (a) The watch design for Pair 9, and (b) the wrist band for Pair 10. The text below (b) is a translated description of how the device works

\subsection{When participants approach a design problem the solutions they come up with are not very detailed}

In several pairs, key functions or user interactions were not explored in detail. Specifically, pairs did not consider a product's states and how to transition between them, which is the core of interaction design. For example, pair 1 state that their watch should be pre-programmed, but do not specify how or by whom.

A. The watch should be programmed with times for medication, so that patients don't press it.

B. The watch will be programmed according to time...

A. The watch will be programmed with regard to the time on how the patients take their medication, so every time when it's time for medication the watch will ring... if it has been programmed to ring at $1 \mathrm{pm}$, it will only ring at $1 \mathrm{pm}$.

B. The alarm should be programmed to ring for a long time to avoid patient to miss their calls, or to take medication.

A. Yes

B. It will be programmed.

A. It will be programmed on how every patient should take their medication, e.g. morning, day and afternoon, so it means when the doctor gives a patient some medication, he should also give the patient a programmed watch on how to take their medication and to remind them when it is time for medication.

\subsection{Participants generate multiple outline solutions}

Several outline solutions were generated by some pairs, and these were either presented as the final outcome or as ideas generated along the way. For example pair 2 generated two separate products: one based on a watch that is for the elderly, and one based on a cell-phone that is for the young. In reaching this conclusion, they also considered an alarm and a more general system as possible solutions.

Pairs 3, 5, 6, 8 and 12 also generated more than one different outline solution.

\subsection{Participants often change direction, one moment talking about one solution and the next about another solution}

This behaviour is evident at varying levels of detail. For example, pair 3 alternate between two main 
solutions five times in the course of the session. In the end their solution involves education from the hospital and a mobile phone alarm. At a finer level of detail, six of the pairs focused on only one main solution, but frequent change of direction between solution elements or initial ideas is evident. This is an excerpt from Pair 10, just before they settle on bracelet as their main solution suggestion:

B. The product should be a beep sound to alert patients about their medication times. If 10 o'clock is the medication time then a "beep" sound will be produced to alert the patient. I don't know how we are going to cater of the deaf patients.

A. We will use a vibrator for deaf patients. It should be programmed like a mobile-phone with offers a vibration mode when you set the alarm like when it rings it should also vibrate.

B. Deaf patients can't hear the alarm.

A. A deaf patient can't hear but other body parts are fully functional. I mean it will awaken body to respond when it vibrates, ok.

B. Then how are we going to design it?

A.I was thinking of a mobile-phone as first priority or may be something like a bracelet. Something regularly used.

B. That should always be worn on the wrist.

A. Always worn on a wrist but people...

B. Sort of a watch....

A. Something that won't differentiate you from other people.

In the end they choose a bracelet that includes an alarm setting to ring and vibrate.

\subsection{Participants use analogy to help set their design direction}

The example below shows how the design focus moves once an analogy is introduced. Pair 2 introduce an alarm analogy which they discuss in the context of deaf and older patients, and use it to set their design direction.

B. Then we should design something like an alarm.

A. An alarm!

B. An alarm can be set to remind some patients. If patients are pensioners, then an alarm might not be that effective due to their slight deafness.

A. Yes

B. I now have no idea as of how to counter dealing with deaf or blind patients. You know how old age ...

A. Pensioners are mostly affected by such problems as slight deafness and partial sight... I think that an alarm is a good idea even though it might difficult for patients with some impairment... I don't know if it's possible to make an alarm to...

A. How are we going to make sure that an alarm is usable to deaf patients? How about blind patients?

$B$. Does this mean that we can introduce objects with audio to cater for patients with any impairment?

B. There will be demonstrating a patient setting an alarm and continue with their daily... till the alarm rings to remind.

A. As reminder.

A. Where is the alarm going to be ringing from? Will it be within another gadget like a television or a watch? $\mathrm{B}$. We are looking at the interactive design, isn't it?
A. Yes

B. Then that means we have to design our own product, which would be used as a system for the patients.

A. It would be a system.

In addition, it was noticeable that participants identified solution analogies, i.e. what the new product might be 'like', rather than problem analogies that focused on the problem domain.

\subsection{Participants generate adaptations of simple, practical, off the shelf designs}

Seven of the 13 pairs suggested an adaptation of a watch or clock. Two suggest adaptations to a mobile phone. Pair 3 suggested a visiting volunteer and education for young people about the importance of taking medication on time; Pair 12 also suggested training alongside a timetable and a phone alarm. Pairs 4 and 10 suggest wrist-band or bracelets. Several end up with different suggestions or a set of suggestions that are complementary, e.g. a timetable of medicines, a pre-programmed mobile phone alarm and training via pamphlets, social workers and radio. Indeed one pair explicitly states "Don't think of complex objects. Think of that cell-phone that we use on a daily basis."

\subsection{Overall design strategy}

Of the four pairs who did not start by suggesting a solution, one discussed which kind of prototyping to use (pair 9), two (pairs 1 and 7) started by considering the different kinds of user:

\section{Pair 1}

A. We are talking about patient who stays at home.

A. Yes

A. Patients who stay home are given medication to take at home. It's usually the youth and the elderly.

B. Yes both youth and the elders.

\section{Pair 7}

A. We have to discuss the kind of a product we are going to design.

B. The first important point is to acknowledge that we have different kinds of individuals. Some might be forgetting to take their medication to abundance and some might be due to drunken habits. We have to come up with an object that can be used to remind them and should be easy to use.

A. Just like you have said that we have different kinds of patients and with different disabilities. So we have to come up with a product that can be used by both disabled and able patients.

Pair 13 considered a mixture of which prototype to use and who the users might be. In all four pairs, the first solution was suggested very soon after this short introduction, i.e. within the first two minutes of the design session. Across all pairs there was almost no analysis of the problem at the beginning. However, pairs did use solution suggestions to explore and identify constraints and elements of the context of use (including users). For example, Pair 
3 alternates between two solutions: having volunteers remind patients, and using a mobile phone. The tentative solution of using volunteers is suggested, but then the further constraints around confidentiality and community care are identified:

\section{Pair 3}

B. I think there can be a group of people assigned the task of reminding patients in their homes about medication, like the home based care people who usually go around homes and give medication to those who are seriously ill.

A. Patient and doctor's confidentiality will not be observed if we have such practices. Do you understand?

B. Yes

A. They will be afraid of the community ridicules or discrimination

This leads to an alternative solution - that of using a mobile phone. The pair considers this solution and identifies further constraints, i.e. "We have the cell-phone for alarms but not all the families can afford it."

As another example, Pair 12 re-define the brief to identify a need for education and training through the radio and television so that home-based patients will understand the importance of taking medication on time.

\section{DISCUSSION}

Some of the behaviours identified above have been described as typical of novice designers. For example, using sketches to capture a design rather than to generate or communicate a design (Welch, 1996), and suggesting a solution before exploring the problem space (Chi et al, 1988: overview). However in both of these cases, the results come from investigations into problem-solving.

Conversely, in a study of creativity in design, Kolodner and Wills (1996) found that designers with experience of the domain will suggest solutions that help them to develop their understanding of the problem domain, i.e. they conjecture solutions.

As discussed above, interaction design is an interdisciplinary subject that draws on methods and processes from many fields including software engineering and product design (Rogers et al, 2011). It has been argued that a 'technical craft' view of the field is not sufficient to provide the wide perspectives needed to be successful (Löwgren and Stolterman, 2007) and so we draw on literature across a wide view of design thinking and problemsolving to discuss the findings.

\subsection{Overall design strategy}

Considering the four strategies suggested by Kruger and Cross (2006), there is little evidence of participants following a problem, information or knowledge-driven strategy, despite the Interaction Design module being structured around a problem- driven approach. Throughout their discussions, knowledge of the users and their context of use is evident, but not explicitly or rigorously examined, as you would expect in a problem-driven approach.

However there is a core of behaviours identified above that indicate a solution-driven overall design strategy. These are: suggesting a solution straight away (behaviour 1), developing solutions that are not very detailed (behaviour 3 ), generating multiple outline solutions (behaviour 4), and often changing direction to consider one solution and then the next (behaviour 5). The solution-driven approach is described as: "the designer focuses on generating solutions, and only gathers information that is needed to further develop a solution. The emphasis lies on generating solutions, and little time is spent on defining the problem, which may be reframed to suit an emerging solution" (Kruger and Cross, 2006: 534). Participants did emphasise generating solutions, and in some cases reframed the problem to suit an emerging solution, e.g. recasting the medication reminder task as a training and education issue.

Kruger and Cross (2006) provide evidence that a solution-driven approach results in higher creativity of designs, although they also found some evidence that it resulted in lower overall quality of designs.

It is possible that our participants were encouraged to take a solution-driven approach to design while still at school as in Botswana, many pupils have design education of some kind in primary and high school. To investigate this option we surveyed 37 local design teachers in Gaborone and asked them whether they had seen this set of behaviours and whether they would encourage or discourage them. The results are in Table 2. This data shows that local design teachers would not universally encourage nor discourage behaviours that support a solution-driven strategy.

Table 2 Local design teachers' reactions to identified behaviours (note that the encourage/discourage response was returned as a group)

\begin{tabular}{|c|c|c|c|}
\hline Behaviour & $\begin{array}{c}\text { Seen by } \\
(\mathbf{n = 3 7 )}\end{array}$ & \multicolumn{2}{|c|}{$\begin{array}{c}\text { Reaction } \\
(\mathbf{n}=\mathbf{7})\end{array}$} \\
\cline { 3 - 4 } & & Encourage & Discourage \\
\hline Solution first & 29 & 3 & 4 \\
\hline $\begin{array}{c}\text { Designs not } \\
\text { detailed }\end{array}$ & 34 & 3 & 4 \\
\hline $\begin{array}{c}\text { Many outline } \\
\text { solutions }\end{array}$ & 35 & 6 & 1 \\
\hline $\begin{array}{c}\text { Often change } \\
\text { direction }\end{array}$ & 37 & 1 & 6 \\
\hline
\end{tabular}

So why did our participants take a solution-driven approach? Nisbett et al (2001) present the case for cognitive styles that are culturally determined and show how these can affect cognitive activities such as how objects are classified, what people attend to, what is judged as salient and how arguments and contradictions are responded to, amongst many other things. However, Nisbett only 
considered US Americans and South East Asians. No work has been carried out to determine cognitive styles in Botswana. Our findings point to the need for investigations into cognitive styles in Botswana.

\subsection{Participants use design methods to represent (capture) design solutions}

Participants will have been taught two different types of design method through the module: textbased methods such as scenarios, and sketchbased methods such as storyboards. The purpose of these methods is to support novice designers in considering the product, its use and its environment. Externalising ideas in this way helps work through the detail and consequences of design solutions.

The transcripts show that pairs spend time discussing design solutions and exploring different scenarios of use, before committing anything to paper. They are therefore externalising ideas through their verbal interactions, rather than through the design methods.

Truong et al (2006) found that novices were concerned about creating sketches as they did not know what to draw and they were concerned about their artistic talents. Either of these factors may have inhibited our participants, and indeed there is evidence in the transcripts to support this view, e.g. "what can we draw?" "I don't know how to sketch?" etc. For experienced designers, sketching is a way of shaping ideas (Fallman, 2003), but using sketches to capture a design rather than to generate or communicate a design has been identified as a novice behaviour (Welch, 1996).

It seems likely that this behaviour is a result of the context of the study, i.e. that participants are in pairs, and feeling inhibited about externalising their ideas, which is a recognised novice behaviour.

\subsection{Participants use analogy to help set their design direction}

Analogy and metaphors are important elements of interaction design - specifically the use of interface metaphors to provide a familiar interface to the users (Rogers et al, 2011). Participants did not use analogy in this way, however, they used it to produce a new design, i.e. to say that a new product will be 'like' another. This design strategy is mentioned in the interaction design module participants study but it is not emphasized.

Ball et al (2004) studied the use of analogies in problem-solving design by novices and experts. They found that novices focused more on concrete existing designs to directly solve the current problem while experts focused on more abstract structural elements of the problem space. The concrete analogical reasoning observed by Ball et al was not used to change the design direction but to suggest direct solutions. Our participants' use of analogy was not sophisticated, but was also not simply a matter of suggesting a direct solution. Rather it was to set the direction for evolving a suitable solution.

\subsection{Participants generate adaptations of simple, practical, off the shelf designs}

Using existing designs as a starting point for new designs is a common and successful practice for initial design thinking. In addition, this behaviour reflects findings in the product innovation literature focusing on concept and prototype development in rural areas of developing countries (referred to as 'base of the pyramid', or BoP markets). For example, Viswanathan and Sridharan (2012) presented 11 product development insights from 13-year long projects in India. One of these propositions was to use existing products for new purposes; they emphasise "the very important role of existing products already possessed and used by BoP consumers in serving as vehicles for addon innovations" ( $p 65)$. Other propositions also emphasize the need to leverage existing infrastructure, and for each product to have multiple purposes.

\section{LIMITATIONS}

The behaviours identified may be a consequence of the constrained task environment. For example, the lack of design details may be an effect of time pressure, although participants commented that they had finished the task set rather than running out of time.

The fact that participants were paired will also have affected the design process. The nature of constructive interaction means that participants explain their thoughts, intentions and actions to each other. Each participant will start from a different frame of reference and what is obvious to one will not be so to the other. This leads to disagreements. These disagreements are similar to reflections, and so reflection would be expected to be more prevalent. Any increase in reflection therefore brought about by our method may imply a design solution or process which is closer to that of a more skilled designer. In short, we may expect the process of constructive interaction to suggest that our participants are more skilled than they are. However participants' reflection was hampered by lack of detail, and so did not approach that expected from a skilled designer.

The constrained task was necessarily short with little scope for complexity in understanding. This may have resulted in the participants being able to rely on 'naïve' design (Dorst, 2003: p83) without needing to call on any sophisticated design behaviour. However, the constrained task problem 
area of home-based medical care has scope for complexity and creativity.

Although we gave the facilitators booklets of instructions, emphasized that they should follow them precisely, and monitored their activity, the facilitators were untrained in running this kind of study and hence may have influenced the results.

The translation from Setswana and Kalanga may have interfered with our understanding on two counts: poor translation and language subtleties being missed. The first was alleviated through the use of more than one translator. The second was alleviated in two ways. Firstly, the research team and the translation team discussed any areas of difficulty or subtlety in translation. Secondly, the research team reviewed the literature on the effects of language on thought processes. This review showed that colour, spatial concepts and gender are the main language influences on thought (Deutscher, 2011). However these concepts did not feature in the protocols.

\section{CONCLUSIONS AND FUTURE WORK}

This paper presents a rare protocol study of novice interaction design behaviour. Despite being taught a problem-driven approach to interaction design, the dominant approach taken by all participant pairs was a solution-driven one.

A 'solution-first' approach has been characterised as a novice problem-solving behaviour, but interaction design is not just about problem-solving, and solution-driven design is an accepted design strategy that can lead to more creative solutions.

So what are the implications of these findings for education? The findings here emphasise the significance of student behaviour as well as curriculum content, and they prompt the question: Do cultural influences lead designers towards a solution-driven approach? This investigation is not able to answer this question on its own, but the findings add to the current debate about $\mathrm{HCl}$ education around the world (Abdelnour-Nocera et al, 2012).

The major element of future work will be to analyse other sets of data we have collected. First, we conducted a diary study with a subset of these participants, and this will enable us to investigate whether the behaviours are different without the constrained task conditions. Second, we conducted the same studies with UK participants who studied the same module as their African counterparts, to see whether behaviour patterns are similar.

One significant question faced by all design educators and mentors is whether novices benefit from being given a structured process to follow at all (Cross, 2007). The discussion is ongoing and there is support for both more structure and less structure. The findings presented here indicate that novice designers may behave in a way that is counter to the structured process they have been taught anyway!

\section{ACKNOWLEDGEMENTS}

This research is funded by the Leverhulme Trust. We would like to thank all our participants, and staff members of Botho University, Francistown for their support in running the studies and translating the protocols.

\section{REFERENCES}

Abdelnour-Nocera, J., Michaelides, M., Austin, A. and Modi, S. (2012) 'An Intercultural Study of $\mathrm{HCl}$ Education Experience and Representation' in Proceedings of ICIC'12, March 21-23, Bengaluru.

Ball, L.J., Ormerod, T.C., and Morley, N.J. (2004) 'Spontaneous analogising in engineering design: a comparative analysis of experts and novices', Design Studies, 25, 495-508.

Blyth, R., Schadewitz, N., Sharp, H., Woodroffe, M., Rajah, D., \& Turugare, R. (2012) A frame signature matrix for analysing and comparing interaction design behavior, In Proceedings of BCS $\mathrm{HCl} 2012$.

Chavan,A. (2005) Another culture, another method. In HCll 2005, New Jersey; Lawrence Erlbaum

Chi, M. T. H., Glaser, R., \& Farr, M. J. (1988). The nature of expertise. Hillsdale, NJ: Erlbaum.

Christensen, B. and Schunn, C. (2007) 'The relationship of analogical distance to analogical function and preinventive structure: the case of engineering design', Memory \& Cognition, 35, 1, 29-38, Springer New York

Clemmensen, T., Hertzum, M., Hornbaek, K., Shi, Q., \& Yammiyavar, P. (2008) Cultural Cognition in the Thinking-Aloud Method for Usability Evaluation. Twenty Ninth International Conference on Information Systems. Paris, 2008, Paper 189

Coley, F., Houseman, O. and Roy, R. (2007) 'An introduction to capturing and understanding the cognitive behaviour of design engineers ', Journal of Engineering Design, vol. 18, no. 4, pp. 311-325.

Cross, N. (2004) 'Expertise in Design: an overview', Design Studies, 25, 427-441.

Cross, N (2007) Designerly ways of knowing. New York: Birkheuser

Deutscher, G. (2011) Through the Language Glass: why the world looks different in other languages, Arrow Books.

Dorst, K. (2003) Understanding Design, BIS Publishers.

Faiola, A., and Matei, S.A. (2005) 'Cultural cognitive style and web design: Beyond a behavioral inquiry into computer-mediated 
communication', Journal of Computer-Mediated Communication.

Fallman, D. (2003) 'Design-oriented HumanComputer Interaction', Proceedings of CHI 2003.

Fendler, J. and Winschiers-Theophilus, H. (2010) 'Towards Contextualised Software Engineering Education: An African Perspective' in Proceedings of ICSE 2010, Cape Town, South Africa, 2-8 May 2010, pp 599-608.

Gautam, V. and Blessing, L. (2010) How Cultural Characteristics Influence Design Processes - an Empirical Study, American Society of Mechanical Engineers.

Gero, J.S. and Tang, H. (2001) 'The differences between retrospective and concurrent protocols in revealing the process-oriented aspects of the design process ', Design Studies, vol. 22, no. 3, pp. 283-295

Kolodner, J.L. and Wills, L. M. (1996) 'Powers of observation in creative design', Design Studies, 17(4), 385-416

Kruger, C. and Cross, N. (2006) 'Solution driven versus problem driven design: strategies and outcomes' in Design Studies 27 527-548.

Kuusela, H. and Paul, P. (2000) 'A Comparison of Concurrent and Retrospective Verbal Protocol Analysis ', The American Journal of Psychology, vol. 113, no. 3, pp. 387-404.

Löwgren, J. and Stolterman, E. (2007) Thoughtful Interaction Design, The MIT Press.

Lotz, N., Sharp, H., Blyth, R., Woodroffe, M., Rajah, D. and Turugare, R. (2013) 'Co-evolving problems and solutions: the case of novice interaction designers in Botswana and the UK' in Proceedings of IASDR.

Maolosi, R., Popovic, V., \& Hickling-Hudson, A. (2007) 'Product analysis based on Botswana's postcolonial sociocultural perspective', International Journal of Design, 1(2), 35-43.

Marsden, G., Maunder, A., and Parker, M. (2008) 'People are people, but technology is not technology', Philosophical Transactions of the Royal Society 366, 3795-3804.

McDonnell, J., \& Lloyd, P. (eds.) (2009) About: Designing Analysing Design Meetings, Taylor and Francis Group, London

Miyake, N. (1986) Constructive interaction and the iterative process of understanding. Cognitive Science 10(2) pp. 151-177

Nisbett, R.E., Peng, K., Choi, I. and Norenzayan, A. (2001) 'Culture and systems of thought: Holistic versus analytic cognition', Psychological review, vol. 108 , no. 2, pp. 291

O'Malley, C.E., Draper, S.W., and Riley, M.S.(1985) Constructive Interaction: A Method for Studying Human-Computer Interaction. In Proceedings of Human-Computer Interaction (INTERACT '84), pp. 269-274 London; Elsevier
Oyugi, C., Dunckley, L., and Smith, A. (2008) 'Evaluation methods and cultural differences: studies across three continents', In Proceedings of NordiCHI08, vol. 358. ACM, New York, pp 318-325. Razzaghi, M, Ramirez, M. and Zehner, R. (2009) 'Cultural patterns in product design ideas: comparisons between australian and iranian student concepts', Design Studies, 30(4) 438-461.

Rogers, Y, Sharp H. and Preece, J. (2011) Interaction Design: beyond $\mathrm{HCl}, 3^{\text {rd }}$ ed, John Wiley Saffer, D. (2010) Designing for interaction: creating smart applications and clever devices $\left(2^{\text {nd }}\right.$ ed), New Riders.

Schön, D.A. (1983) The reflective practitioner, Basic Books, New York.

Sharp H. Rogers, Y, and Preece, J. (2007) Interaction Design: beyond $\mathrm{HCl}, 2^{\text {nd }}$ ed, John Wiley Smith, A., Bannon, L. and Gulliksen, J. (2010) 'Localising $\mathrm{HCl}$ Practice for Local Needs' in Proceedings of India HCl 2010/ Interaction Design \& International Development 201020 - 24 March 2010 Indian Institute of Technology, Bombay, Mumbai, India.

Smith, A., Joshi, A., Liu, Z., Bannon, L., Gulliksen, $\mathrm{J}$. and $\mathrm{Li}, \mathrm{C}$. (2007) Institutionalising $\mathrm{HCl}$ in Asia, Interact 2007, pp85-99.

Truong, K.N., Hayes, G.R. and Abowd, G.D. (2006) Storyboarding: An Empirical Determination of Best Practices and Effective Guidelines, in Proceedings of DIS 2006, June 26-28, 2006, University Park, Pennsylvania, USA.12-21

Valkenburg, R. and Dorst, K. (1998) 'The reflective practice of design teams' Design Studies, 19(3), 249-271

Van den Haak, M.J., de Jong, M.D.T. and Schellens, P.J. (2004) 'Employing think-aloud protocols and constructive interaction to test the usability of online library catalogues: a methodological comparison', Interacting with Computers, 16(6), pp. 1153-1170

Visser, W. (2006) 'Designing as construction of representations: A dynamic viewpoint in cognitive design research' Human-Computer Interaction, 21(1), 103.

Viswanathan, M. and Sridharan, S. (2012) 'Product Development for the BoP: Insights on Concept and Prototype Development from University-Based Student Projects in India' Journal of Product Innovation Management, 29(1), 52-69.

Welch, M. (1996) The Strategies Used by Ten Grade 7 Students, Working in Single-Sex Dyads, to Solve a Technological Problem, Unpublished doctoral dissertation, McGill University, Montreal 